\title{
Correction to: Injuries in Field Hockey Players: A Systematic Review
}

\author{
Saulo Delfino Barboza ${ }^{1}$ Corey $\mathrm{Joseph}^{2} \cdot \mathrm{Joske} \mathrm{Nauta}^{1} \cdot$ Willem van Mechelen ${ }^{1,3,4,5}$. \\ Evert Verhagen ${ }^{1,4,6}$
}

Published online: 14 February 2018

(C) Springer International Publishing AG, part of Springer Nature 2018

\section{Correction to: Sports Med https://doi.org/10.1007/s40279-017-0839-3}

An Online First version of this article was made available online at https://link.springer.com/article/10.1007/s40279017-0839-3 on 3 January 2018. An error was subsequently identified in the article, and the following correction should be noted:

Page 1: The listing of the author names and affiliations, which previously read:

The original article can be found online at https://doi.org/10.1007/ s40279-017-0839-3.

Evert Verhagen

e.verhagen@vumc.nl

1 Amsterdam Collaboration on Health and Safety in Sports, Department of Public and Occupational Health, Amsterdam Public Health Research Institute, VU University Medical Center, Van der Boechorststraat 7, 1081 BT Amsterdam, The Netherlands

2 Monash Health, Clayton, Australia

3 School of Human Movement and Nutrition Sciences, Faculty of Health and Behavioural Sciences, University of Queensland, Brisbane, QLD 4072, Australia

4 Division of Exercise Science and Sports Medicine, Department of Human Biology, Faculty of Health Sciences, University of Cape Town, Anzio Road, Observatory 7925, Cape Town, South Africa

5 School of Public Health, Physiotherapy and Population Sciences, University College Dublin, Belfield, Dublin 4, Ireland

6 Australian Collaboration for Research into Injury in Sport and its Prevention, Federation University Australia, Lydird Street South, Ballarat, VIC 3350, Australia
Saulo Delfino Barboza ${ }^{1} \cdot$ Corey Joseph ${ }^{2} \cdot$ Joske Nauta $^{1}$. Willem van Mechelen ${ }^{1,3,4,5}$. Evert Verhagen ${ }^{1,2,4}$

${ }^{1}$ Amsterdam Collaboration on Health and Safety in Sports, Department of Public and Occupational Health, Amsterdam Public Health Research Institute, VU University Medical Center, Van der Boechorststraat 7, 1081 BT Amsterdam, The Netherlands

${ }^{2}$ Australian Collaboration for Research into Injury in Sport and its Prevention, Federation University Australia, Lydird Street South, Ballarat, VIC 3350, Australia

${ }^{3}$ School of Human Movement and Nutrition Sciences, Faculty of Health and Behavioural Sciences, University of Queensland, Brisbane, QLD 4072, Australia

${ }^{4}$ Division of Exercise Science and Sports Medicine, Department of Human Biology, Faculty of Health Sciences, University of Cape Town, Anzio Road, Observatory 7925, Cape Town, South Africa

${ }^{5}$ School of Public Health, Physiotherapy and Population Sciences, University College Dublin, Belfield, Dublin 4, Ireland

Should read:

Saulo Delfino Barboza ${ }^{1}$. Corey Joseph ${ }^{2}$. Joske Nauta ${ }^{1}$. Willem van Mechelen ${ }^{1,3,4,5}$. Evert Verhagen ${ }^{1,4,6}$

${ }^{1}$ Amsterdam Collaboration on Health and Safety in Sports, Department of Public and Occupational Health, Amsterdam Public Health Research Institute, VU University Medical Center, Van der Boechorststraat 7, 1081 BT Amsterdam, The Netherlands

${ }^{2}$ Monash Health, Clayton, Australia

${ }^{3}$ School of Human Movement and Nutrition Sciences, Faculty of Health and Behavioural Sciences, University of Queensland, Brisbane, QLD 4072, Australia

${ }^{4}$ Division of Exercise Science and Sports Medicine, Department of Human Biology, Faculty of Health 
Sciences, University of Cape Town, Anzio Road, Observatory 7925, Cape Town, South Africa

${ }^{5}$ School of Public Health, Physiotherapy and Population Sciences, University College Dublin, Belfield, Dublin 4, Ireland
${ }^{6}$ Australian Collaboration for Research into Injury in Sport and its Prevention, Federation University Australia, Lydird Street South, Ballarat, VIC 3350, Australia 